\title{
Editorial - Volume 15, Issue Number 3
}

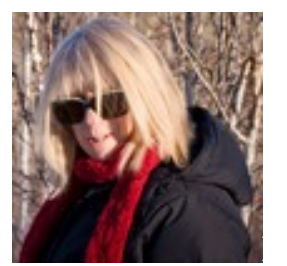

Dianne Conrad

Co-Editor, IRRODL

Your early summer reading is here! Or is it?! Clearly, for an international journal, this seasonal reference represents a Western/ Northern-based sentiment. Perhaps that's just one small illustration of the scope and accompanying challenges of making sense of the diverse contributions that IRRODL attracts.

In this issue of more than 15 articles, we once again present to you the range of research that you have come to expect from us - research that spans the world and many aspects of the ODL field. What *don't* we have in this issue? There is nothing on OER this time around; however, watch for an upcoming special issue that will feature current research in this quickly developing area.

This issue features several pieces that highlight the business of successful teaching, faculty-learner and learner-learner interaction. From Serbia, Raspopovic, Jankulovic, Runic, and Lucic examine, in a case study, success factors in e-learning, from the perspective of a developing country in transition from traditional modes of learning to technology-enhanced modes of learning. Similarly, Mbatha gives us a case study that considers global transition in higher education as the University of South Africa transitions from a traditional model of learning to a new socially mediated model. And in another case study, Sadykova examines mediating knowledge through peer interaction in a multicultural online course offered in the US.

Samuels-Peretz considers the nature of learners' interactions with others, also at an American institution, with a special emphasis on gendered knowing, using Belenky, Clinchy et al.'s 1986 seminal study that produced Women's Ways of Knowing. Chang, Shen, and Liu explore the faculty role in online instruction while $\mathrm{Vu}, \mathrm{CaO}, \mathrm{Vu}$, and Cepero researched success factors for learners in an online professional development course. Clearly, we are still intrigued, internationally, with what makes online teaching and learning successful. How can we make it better? Borup, West, Thomas, and Graham suggest, in their piece on the impact on students of video feedback, that the resultant learner-instructor connection is enriched. Video use is also the topic of 
Lujubojevic, Vaskovic, Stankovic, and Vaskovic's research on its efficacy as a tool for increasing the quality of experience (QoE) in multimedia instruction.

Social environments and personal learning environments continue to also pique our research interest. Diaz, Martinez, and McMullin, in a Spanish study, put forward the suggestion that social networks can be transformed into learning communities, into rich environments that meet many learner needs. Also looking at ways to enhance online learning environments, Alden Rivers, Richardson, and Price, in a British study, analyzed educators' own perspectives to determine what is needed to create a more fertile ground for reflective learning.

In more specific areas of our field, two articles deal with mobile learning. Huang, Hsiao, Tang, and Lien, exploring mobile learning, contend that language learning success lies not only in technology-supported learning activities, but also in consecutive learning opportunities. And in another piece that considers the interplay of language learning with mobile technology, Young and Hung researched open courseware at National Tsing Hua University (NTHU), concluding that support for the use of mobile learning is essential for learner motivation and success, and for the continued growth of accessible learning opportunities.

But there's more! Ching examines the use of role-play in peer feedback using VoiceThread technology as an example of constructivist learning. Tustin, presenting another South African perspective, considers the advantages of telecommuting for academic performance in that country's open and distance learning culture.

Lastly, this super summer issue offers reading in the Technical Notes and Leadership Notes sections. Suen's piece on the sticky business of MOOC assessment is not only interesting but also serves as a good example of what a "note" can look like in IRRODL; it's a handy publication vehicle for items that are not classified as research articles. And from Athabasca University students, complementing the two articles on mobile and language learning, another "notes" piece outlines, from a leadership perspective, a pilot project on mobile language learning. IRRODL's notes sections offer good publication opportunities for in-progress or formative research initiatives. Consider submitting to this venue!

\section{Athabasca University $\mathbf{a}$}

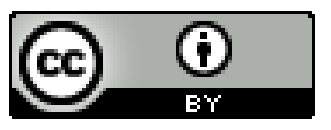

